\title{
BOSC: A Better Oscillation Detection Method, Extracts Both Sustained and Transient Rhythms from Rat Hippocampal Recordings
}

\author{
Adam M. Hughes, ${ }^{1}$ Tara A. Whitten, ${ }^{2}$ Jeremy B. Caplan, ${ }^{1,2}$ and Clayton T. Dickson ${ }^{1,2,3 *}$
}

\begin{abstract}
Neuronal population oscillations at a variety of frequencies can be readily seen in electroencephalographic (EEG) as well as local field potential recordings in many different species. Although these brain rhythms have been studied for many years, the methods for identifying discrete oscillatory epochs are still widely variable across studies. The "better oscillation detection" (BOSC) method applies standardized criteria to detect runs of "true" oscillatory activity and rejects transient events that do not reflect actual rhythms. It does so by estimating the background spectrum of the actual signal to derive detection criteria that include both power and duration thresholds. This method has not yet been applied to nonhuman data. Here, we test the BOSC method on two important rat hippocampal oscillatory signals, the theta rhythm and slow oscillation (SO), two large amplitude and mutually exclusive states. The BOSC method detected both the relatively sustained theta rhythm and the relatively transient SO apparent under urethane anesthesia and was relatively resilient to spectral features that changed across states, complementing previous findings for human EEG. Detection of oscillatory activity using the BOSC method (but not more traditional Fourier transform-based power analysis) corresponded well with human expert ratings. Moreover, for near-continuous theta, BOSC proved useful for detecting discrete disruptions that were associated with sudden and large amplitude phase shifts of the ongoing rhythm. Thus, the BOSC method accurately extracts oscillatory and nonoscillatory episodes from field potential recordings and produces systematic, objective, and consistent results—not only across frequencies, brain regions, tasks, and waking states, as shown previously, but also across species and for both sustained and transient rhythms. Thus, the BOSC method will facilitate more direct comparisons of oscillatory brain activity across all types of experimental paradigms. $\odot 2011$ Wiley Periodicals, Inc.
\end{abstract}

KEY WORDS: theta; slow oscillation; urethane anesthesia; brain state; spectral analysis

\section{INTRODUCTION}

Neuronal oscillations arise from the intrinsic properties of neurons and their functional network interactions. Population oscillations at

\footnotetext{
${ }^{1}$ Department of Psychology, University of Alberta, Edmonton, Alberta, Canada; ${ }^{2}$ Centre for Neuroscience, University of Alberta, Edmonton, Alberta, Canada; ${ }^{3}$ Department of Physiology, University of Alberta, Edmonton, Alberta, Canada

Grant sponsors: Natural Sciences and Engineering Research Council of Canada (NSERC), Alberta Ingenuity Fund (AIF), Alberta Heritage Foundation for Medical Research (AHFMR). Grant numbers: NSERC, 249861, 341662; AIF, 200800568. CTD is a AHFMR Senior Scholar.

*Correspondence to: Clayton T. Dickson, Ph.D., Department of Psychology, University of Alberta, Biological Sciences Building, P217, Edmonton, Alberta, Canada T6G 2E9. E-mail: clayton.dickson@ualberta.ca

Accepted for publication 12 August 2011

DOI 10.1002/hipo.20979

Published online 13 October 2011 in Wiley Online Library (wileyonlinelibrary.com).
}

diverse frequencies can be readily seen in electroencephalographic (EEG) as well as local field potential (LFP) recordings in many different species and have been shown to correlate with ongoing behavior and with the processing of stimuli in the immediate environment in addition to both online and offline mnemonic functions (Berger, 1929; Vanderwolf, 1969; Worden et al., 2000; Bland and Oddie, 2001; Caplan et al., 2001; Buzsaki and Draguhn, 2004; Klimesch et al., 2007; Marshall and Born, 2007; Freunberger et al., 2009; Dickson, 2010). Although oscillations have been studied for many years (Berger, 1929), identifying discrete oscillatory epochs in EEG traces is still quite variable across studies (van Vugt et al., 2007). A major challenge for the majority of current methods is that they are sensitive not only to oscillations but also to sharp transient potentials (either spontaneous or event-related) that are nonoscillatory, as well as artifacts caused by eye movements and static discharges. Another problem is that criteria for oscillation detection require dynamic adjustments across frequency due to the colored-noise background spectrum of field potentials wherein low-frequency components tend to have higher amplitudes than highfrequency components (Schlesinger and West, 1988; Caplan et al., 2001; Buzsaki and Draguhn, 2004). These problems make a systematic study of brain oscillations difficult both within and across different brain states (much less within and across different species), because threshold and statistical criteria tend to vary methodologically as do the background properties of signals.

With these pitfalls in mind, Caplan et al., (2001) designed the "better oscillation detection" (BOSC) method. The method calculates a power threshold that is derived from an estimate of the local background spectrum and a duration threshold that is scaled to each specific frequency (Fig. 1). This method detects only segments of recorded activity that meet these rigorous criteria and rejects transient events that are not oscillatory but that can create spectral peaks or changes in power that can be erroneously interpreted as rhythmic activity. These properties make BOSC a principled and objective method that could place different studies on the same footing and reduce researcher subjectivity.

At present, the BOSC method has only been applied to detect sporadic oscillations in awake, behaving humans (Caplan et al., 2001; Caplan et al., 2003; 


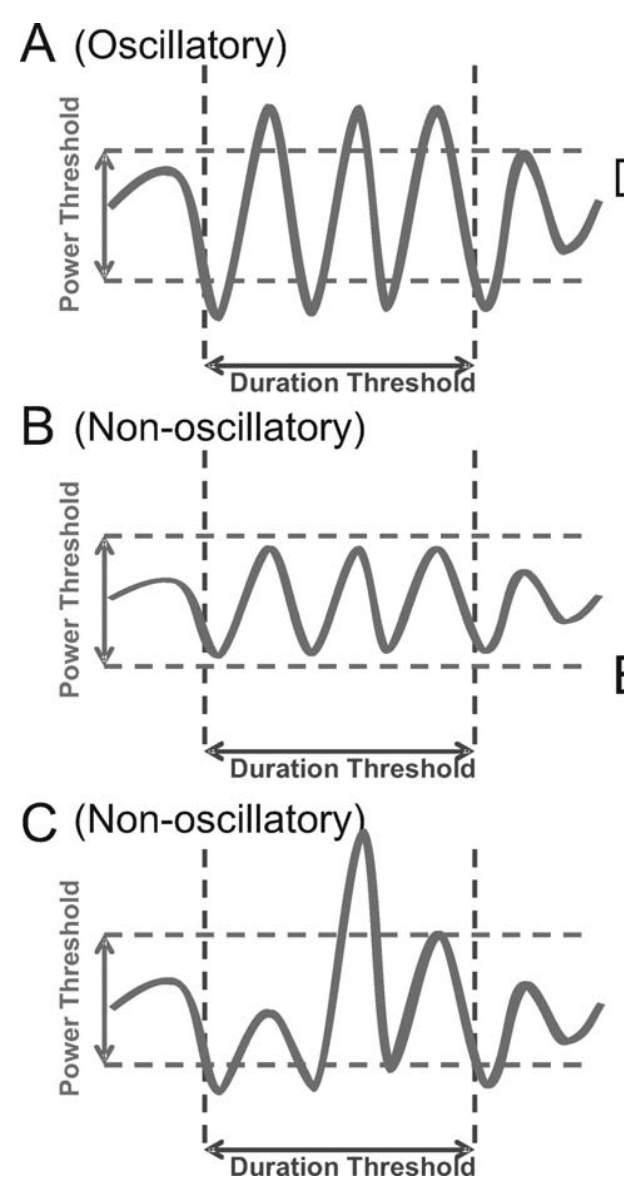

FIGURE 1. Schematic depiction of the BOSC oscillatory episode detection method. A power threshold $\left(P_{\mathrm{T}}\right)$ must be exceeded for a minimum duration of time (i.e., number of cycles), designated by a duration threshold $\left(D_{\mathrm{T}}\right)$. A: A hypothetical example raw trace that exceeds both of these thresholds, and would be counted as oscillatory. B: A hypothetical example of a raw trace that exceeds the duration threshold, but fails to exceed the power threshold, and would thus not be labeled as oscillatory. C: A hypothetical example of a raw trace that exceeds the power threshold, but fails to exceed the duration threshold, and would thus not be labeled as oscillatory.

Ekstrom et al., 2005; Caplan and Glaholt, 2007; Watrous et al., 2011; Whitten et al., 2011) and during sleep (Marzano et al., 2011). Ideally this method would also be useful for LFP recordings obtained from nonhuman animals, especially rats, the chief subjects used to study hippocampal rhythms, both within and across brain state changes such as those that occur during ongoing behaviour, sleep, and also under anesthesia. Hippocampal state alternations are associated with both sporadic and continuous trains of oscillatory activity including theta, ripples, and the slow oscillation (SO; Bland, 1986; Chrobak et al., 2000; Buzsaki, 2002; Wolansky et al., 2006; Clement et al., 2008), and these activities are thought to play important roles in both online and offline memory processing (Buzsaki, 1989; Hasselmo, 2005; Dickson, 2010). However, it is unclear how BOSC would perform for the detection of different types of state-dependent activity, especially when state changes occur within the data segment being analyzed. This is an important
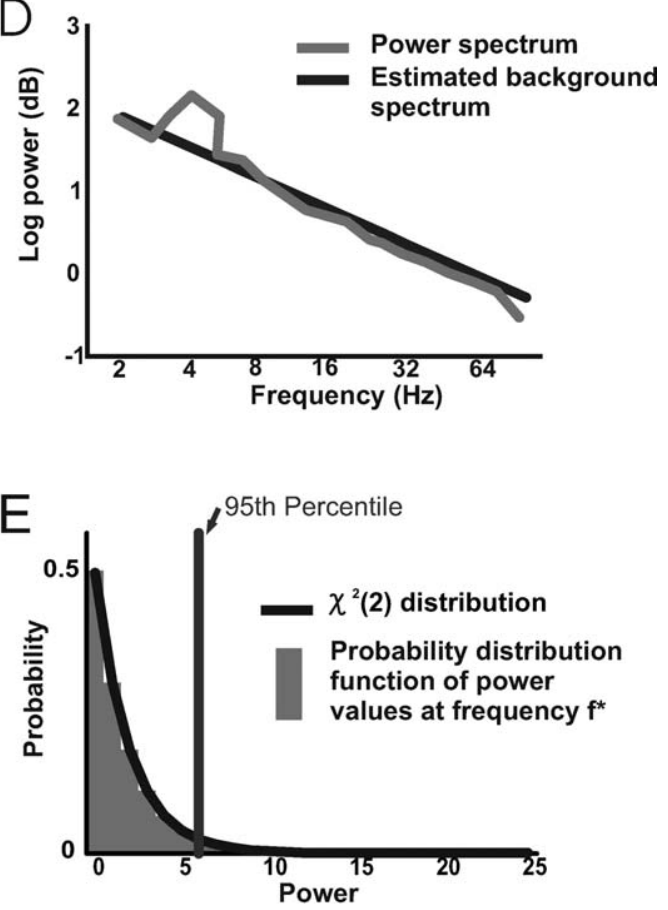

Both thresholds are scaled to the frequency of interest. $D_{\mathrm{T}}$ is a fixed number of cycles, most commonly set to 3. D: A hypothetical example of a power spectrum with a peak potentially reflecting theta oscillations (gray), and the modeling of the background spectrum with a linear regression (black). E: A hypothetical probability distribution function of power values at frequency $f^{*}$ can be fit by a $\chi^{2}(2)$ distribution (see text), which is used to determine the power threshold $\left(P_{\mathrm{T}}\right)$ for oscillation detection, in this case based on the 95th percentile of the $\chi^{2}(2)$ distribution. This figure has been modified from Figure 1 of Whitten et al. (2011).

question, because one of the hallmark features of a state change is an alteration of the overall shape of the power spectrum. If this reflects a change in the background colored-noise power spectrum, the estimate of the background spectrum that the BOSC method relies on may be skewed, and this could undermine its effectiveness in oscillation detection.

To evaluate the robustness of the BOSC method for both continuous and sporadic oscillatory activity across state changes, we tested it on LFPs recorded from the hippocampus of urethane-anesthetized rats. These recordings demonstrate both continuous (theta) and sporadic (SO) rhythmic activity in addition to spontaneous state alternations (Wolansky et al., 2006; Clement et al., 2008). This allowed us to test the ability of the method for both types of activity based on background samples within and across states and to validate detections with those made by human experts using more conventional (but still qualitative) analyses. 


\section{METHODS}

\section{Hippocampal Recordings}

Hippocampal LFPs were recorded as previously described and published (Wolansky et al., 2006). In brief, monopolar Teflon-coated stainless-steel electrodes (bare diameter $125 \mu \mathrm{m}$, A-M systems) were targeted to the hippocampal fissure of the dorsal hippocampus of urethane-anesthetized rats (coordinates from Bregma: AP: -3.3 , ML: $\pm 2.0, \mathrm{DV}:-2.8$ to $-3.3 \mathrm{~mm}$ ). A representative placement is shown in Figure 2A. Recordings (referenced to the stereotaxic apparatus) were amplified at a gain of 1,000, bandpass filtered from 0.1 to $500 \mathrm{~Hz}$ (Model 1700 amplifier: A-M systems), and digitized at $1 \mathrm{kHz}$ (Digidata-Axon Instruments). As we have previously shown (Wolansky et al., 2006; Clement et al., 2008) these recordings demonstrate spontaneous state alternations between activated (theta activity), transition (large amplitude irregular activity, LIA), and deactivated (LIA + SO) states which we could characterize by the observation of the raw traces (Fig. 2A), via Fourier-transform-based spectral methods (Fig. 2B), and by autocorrelation analysis (Fig. 2C).

\section{Fourier Peak Power Analysis of LFPs}

In our previous work, state changes were characterized by extracting and comparing power values at specific bandwidths across epochs using traditional Fourier spectrographic analysis. Specifically, power spectral density estimates were computed on sliding windows of the LFP data using a modified version of Welch's periodogram method implemented in Matlab (versions 5 or 7: Mathworks, Natick, MA; Clement et al., 2008; Schall et al., 2008). In the bottom panels of Figure 2, the spectral power in the theta $(3-4 \mathrm{~Hz})$ and $\mathrm{SO}(0.5-1.5 \mathrm{~Hz})$ bandwidths are shown in panel $\mathrm{E}$ for a windowed analysis of the LFP traces shown in panel $\mathrm{D}$. These windows were $3 \mathrm{~s}$ in length with a $1 / 3$ rd overlap (1 s). To classify states and their transitions, the distribution of the ratios of logged power values of $\mathrm{SO}$ to theta bandwidths was evaluated across time. Values lying in the trough between bimodal peaks of this distribution were used as a threshold for state changes; values above this threshold were considered SO, and values below were considered non-SO. A similar analysis using the ratio of theta over $\mathrm{SO}$ power was used to distinguish theta and not-theta states. Time periods between these two states that were classified as not-theta and not-SO were labeled LIA.

\section{The BOSC Method}

For all computations, a continuous wavelet transform using a Morlet wavelet with width $=6$ was performed on the data. The Morlet wavelet is commonly used for the analysis of human EEG because of its sinusoidal shape and tapering ends. The success of wavelet-based EEG analysis may be attributed to the similarity of the wavelet to the target signal (Schiff et al., 1994). Data analysis was performed using MATLAB 7.6 (see supplementary materials of the work by Whitten et al.,

\section{A}

1) Theta
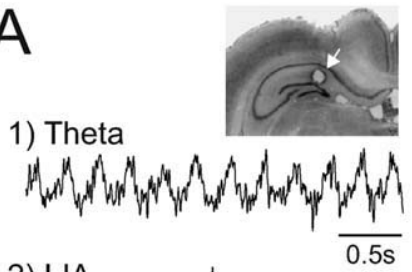

2) LIA

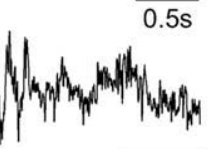

3) $\mathrm{SO}$
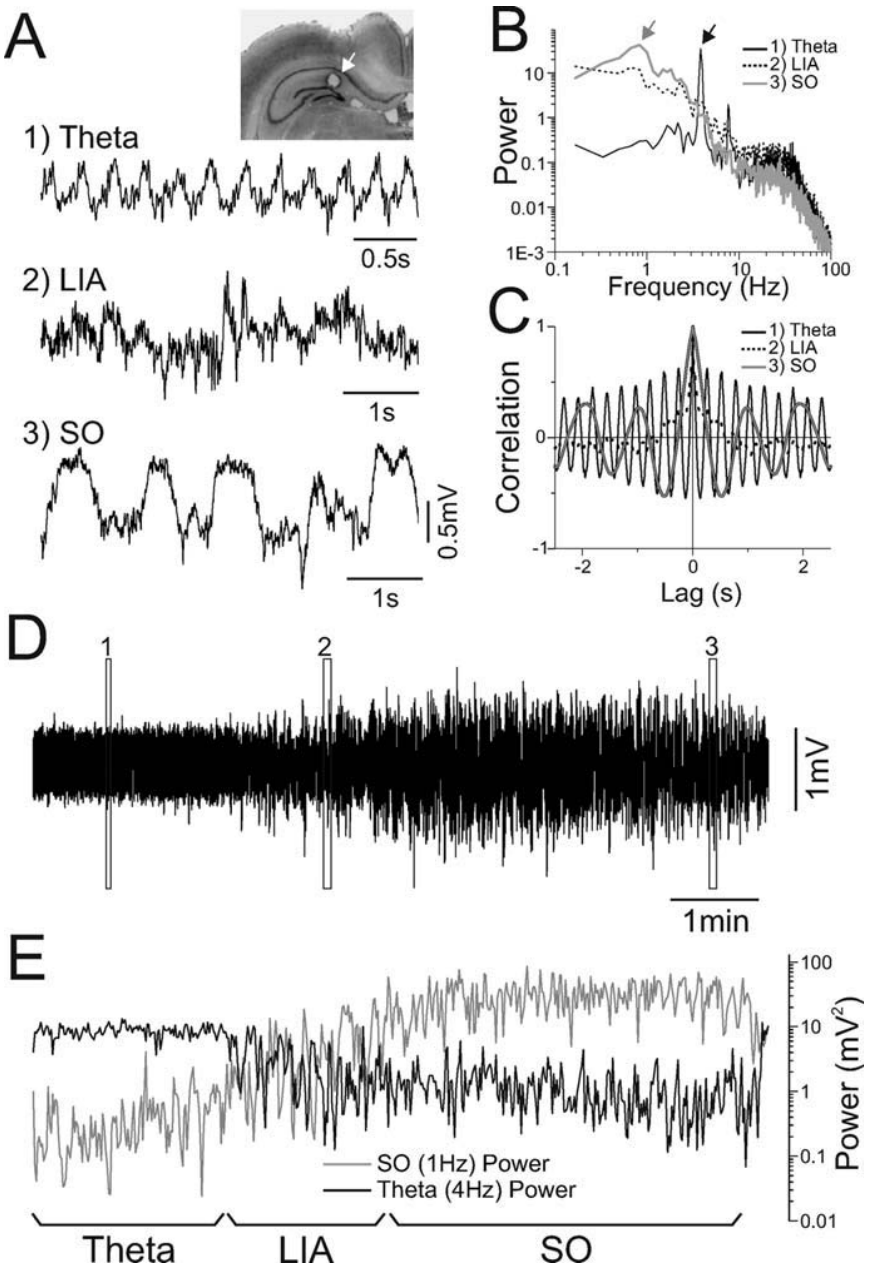

FIGURE 2. Spontaneous hippocampal LFP waveforms and their transforms as recorded in the urethane-anesthetized rat. A: Example traces of each of the three hippocampal states: (1) Theta, (2) large amplitude irregular activity (LIA), and (3) the slow oscillation (SO). Only theta and the SO show oscillatory components. The inset shows the location of a typical recording site in a photomicrograph of the dorsal hippocampus in a coronal section of rat brain. The circular lesion at the hippocampal fissure (white arrow) was made by passing DC current through the electrode tip following the experiment. Each state is also represented in spectral (B) and autocorrelation $(C)$ plots taken from the examples shown in (A). D: A long duration LFP trace from which each trace in A was taken (see numerals corresponding to panel numbers in $\mathrm{A}$ ). $\mathrm{E}$ : Peak power in both theta and SO bandwidths was extracted from the signal shown in $\mathrm{D}$ and plotted on the same time scale. Based on the ratio of the extracted power values, theta, LIA, and SO states could be separated as denoted.

2011). Frequencies were sampled logarithmically from $0.71-$ $76.1 \mathrm{~Hz}$, in 28 frequency steps. Wavelet power spectra, Power $(f)$, were then fit with a linear regression in $\log -\log$ coordinates to estimate the colored-noise background spectrum (Fig. 1D). A power threshold, $P_{\mathrm{T}}$, for each frequency was calculated as the 95th percentile of the theoretical $\chi^{2}(2)$ distribution of wavelet power values (Fig. 1E), assuming the mean background spectrum estimated by the linear regression would be the mean of the corresponding $\chi^{2}(2)$ distribution at a given frequency 
(Caplan et al., 2001; Whitten et al., 2011). The reason the distribution of squared wavelet coefficients is expected to have the form $\chi^{2}(2)$ is that raw LFP signal is approximately Gaussiandistributed; power, the square of the wavelet coefficient, is therefore $\chi^{2}$-distributed (Percival and Walden, 1993). As wavelet coefficients are complex, each wavelet power value is the sum of two squared Gaussian-distributed values (Pythagoras' theorem), one for the real and one for the imaginary part, resulting in a $\chi^{2}$ with $d f=2$. Figures 3 and 4 show the theoretical $\chi^{2}(2)$ plotted over the observed probability distribution functions of wavelet power. For frequencies that are expected to be oscillatory within a given state (Figs. 3D and $4 \mathrm{~B}$ ), the estimated distribution deviates considerably from the observed distribution, especially due to a surplus of high power values. For other (nonrhythmic) frequencies (other panels in Figs. 3 and 4), the expected distributions fit the observed distributions well.

Power values exceeding the power threshold were then subjected to the duration threshold $D_{\mathrm{T}}=3$ cycles. The duration threshold criterion is that $P_{\mathrm{T}}$ must be exceeded for a minimum of $D_{\mathrm{T}}$ cycles $\left(D_{\mathrm{T}} / f\right.$ at a frequency of interest, $\left.f\right)$. Oscillatory epochs were thus considered "detected" when both thresholds were met (Fig. 1A when compared with $1 \mathrm{~B}$ and $1 \mathrm{C}$ ). Note that in the continuous wavelet transform there is considerable overlap between successive wavelet-power values. The degrees of freedom for this evaluation can be computed (i.e., Plett, 2000) and for the example of a $4-\mathrm{Hz}$ rhythm meeting the minimum $D_{\mathrm{T}}$ of three cycles, the independent degrees of freedom are 2.7. Thus, although the $P_{\mathrm{T}}$ sets the initial level of conservatism, $D_{\mathrm{T}}$ makes detection slightly more conservative due to the reduced probability that $P_{\mathrm{T}}$ could be exceeded for $D_{\mathrm{T}}$ cycles.

To assess the performance of the BOSC method and its sensitivity (or resilience) to state changes, signals were analyzed based on background spectrum estimates that used data either from within state or aggregating between states. This tested the influence of state on the tuning of the $P_{\mathrm{T}}(f)$, which could potentially undermine the robustness of the method to detect oscillations consistently despite state changes, which in a realistic research setting, could quite plausibly be unknown.

\section{Comparison of Analysis Methods}

To assess the performance of the Fourier peak power analysis (FPPA) and BOSC methods, we computed the Pearson correlation for the results of the classifications across different methods, including those made by human raters. We focused on $\mathrm{SO}$, because we have previously determined that this activity is more difficult to classify because of its sporadic nature (Wolansky et al., 2006). FPPA and BOSC methods were compared with each other and were each compared with ratings completed by a panel of expert raters (authors AMH, TAW, and CTD). As the initial implementation of FPPA was very poorly correlated with both BOSC and our raters we devised a more conservative version that required at least two adjacent windows (just prior and subsequent) to reach threshold in order for the middle window to be classified as oscillatory.
Each rater evaluated windowed signals from deactivated periods that had been previously analyzed by either the FPPA or BOSC technique. These windowed and band-pass filtered (between 0.5 and $1.5 \mathrm{~Hz}$ ) signals were displayed in a randomized order, along with upper and lower limits, which were calculated at two times the root mean square (RMS) of the filtered signal across the entire epoch. As well, displayed alongside the traces were (1) the autocorrelation of the windowed signal superimposed on an autocorrelation of a $1-\mathrm{Hz}$ sine wave and (2) the power spectral density of the raw, unfiltered signal along with the upper and lower confidence limits for the entire epoch. For each window, reviewers applied their expertise to rate windows as either oscillatory or not at $1 \mathrm{~Hz}$.

\section{RESULTS}

As shown in Figure 2 and as described previously (Wolansky et al., 2006; Clement et al., 2008), the LFP of the rat hippocampus under urethane-anesthesia showed spontaneous state alternations between activated and deactivated patterns. The activated pattern consisted of an almost continuous $3-4 \mathrm{~Hz}$ rhythm (theta), whereas the deactivated pattern was characterized by a broadband irregular signal with higher power at low frequencies (LIA). Also apparent during the deactivated pattern were discrete epochs of sporadic large amplitude $\sim 1 \mathrm{~Hz}$ oscillatory activity (SO). These three types of activity could be differentiated based on their raw waveforms, spectra, and autocorrelation functions as shown in Figures 2A-C.

By extracting power from the Fourier-transform-based spectrographic analysis of signals in both the theta $(3-4 \mathrm{~Hz})$ and SO $(1 \mathrm{~Hz})$ bandwidths and plotting these values as a function of time we could evaluate state changes systematically. Theta power was high during activated states and low during deactivated, whereas SO power showed the opposite pattern. In this, and previous work, we used the power in either peak bandwidth (and/or the ratio) to characterize theta versus $\mathrm{SO}$ states. The gross separation of states using this FPPA method is shown in Figure 2E.

\section{The BOSC Method for Detecting Hippocampal Theta}

Given that the BOSC method has only been used to extract sporadic episodes of oscillatory activity from a recording, we first assessed the ability of this method to detect the $3-4 \mathrm{~Hz}$ theta rhythm during the activated state. As shown in Figures $5 \mathrm{~B}$; C-F, nearly continuous oscillatory theta $(4 \mathrm{~Hz})$ activity was extracted from the activated state. There were some transient breaks observed during the activated state, and a few epochs of $4 \mathrm{~Hz}$ activity were detected during the deactivated state as well. Although the linear regression fit differently depending on which signal was used to estimate the background spectrum (Fig. 5A), especially at the lower frequencies, the detection of $4 \mathrm{~Hz}$ oscillations was nonetheless very similar except when the 

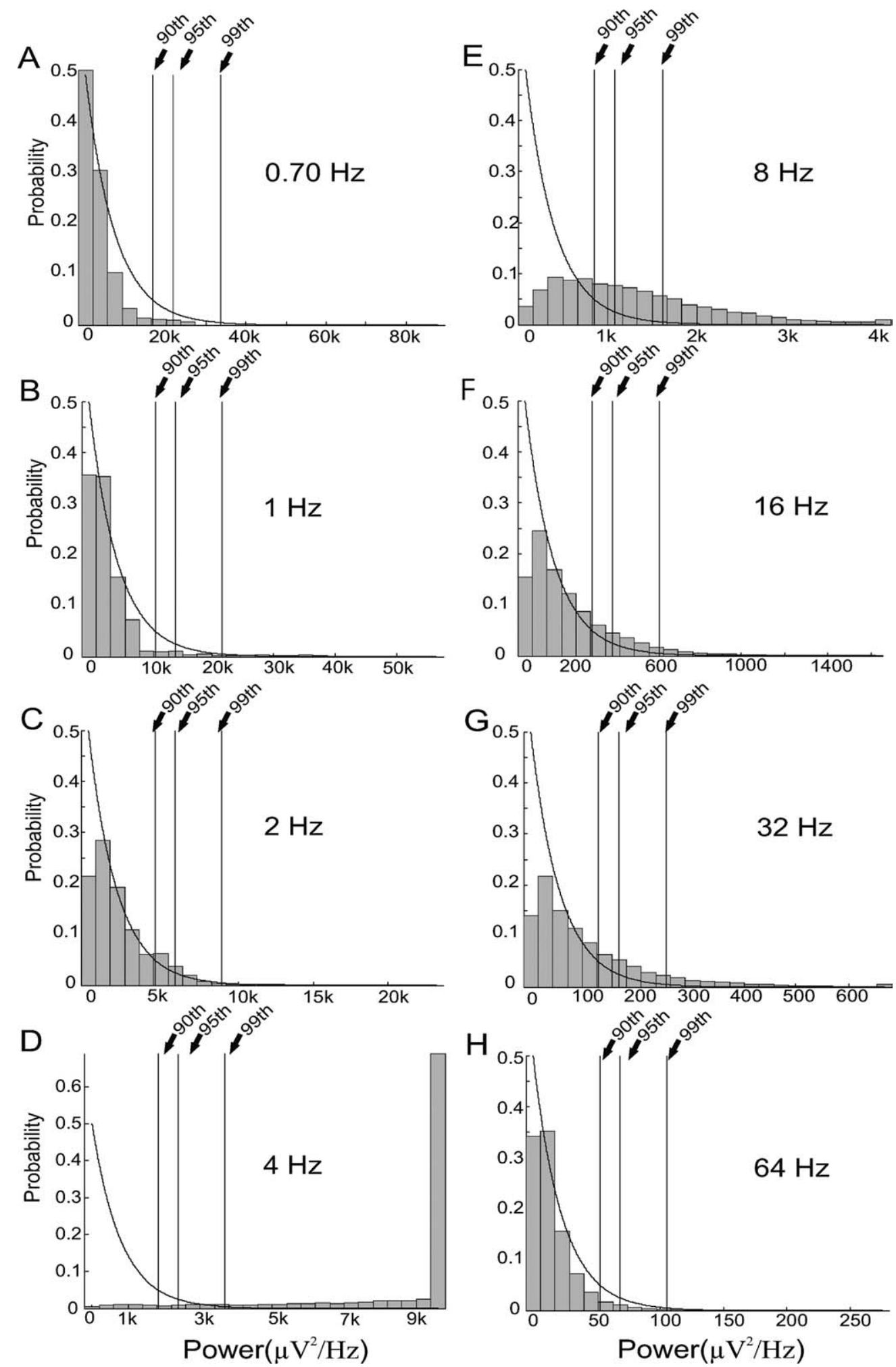

FIGURE 3. Probability distributions of power values for activated states. For all panels, bars represent the empirical probability distribution function of power values from the signal used to estimate the background spectrum in 25 equally spaced bins for the a given frequency. The thin curve represents the theoretical $\chi^{2}(2)$ probability distribution function based on the estimated mean power at the same frequency, derived from the linear-regression fit obtained from

the activated state. Vertical lines represent the 90th, 95th, and 99th percentile thresholds (left to right). Note that for theta frequencies (most prominently at $4 \mathrm{~Hz}$ ), a great proportion of the power values are far beyond the range plotted here; those are summed in the rightmost bin. At theta frequencies, the distributions deviate substantially from the expected $\chi^{2}(2)$ but at other frequencies, the fit is reasonable, in line with the assumptions of the BOSC method. 

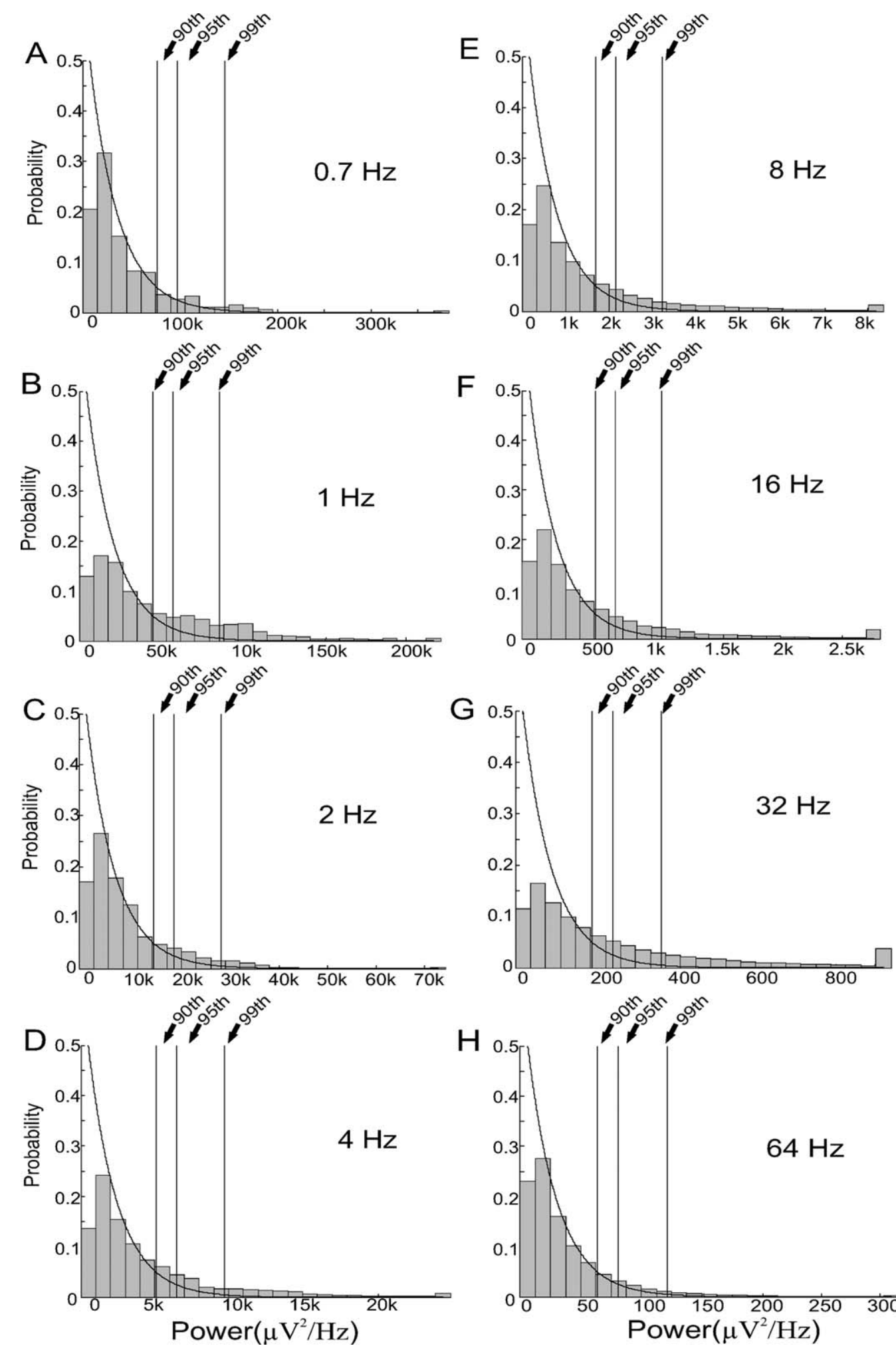

FIGURE 4. Probability distributions of power values for deactivated states. For all panels, bars represent the empirical probability distribution function of power values from the signal used to estimate the background spectrum in 25 equally spaced bins for the given frequency. The thin curve represents the theoretical $\chi^{2}(2)$ probability distribution function based on the estimated mean power at the same frequency, derived from the linear-regres-

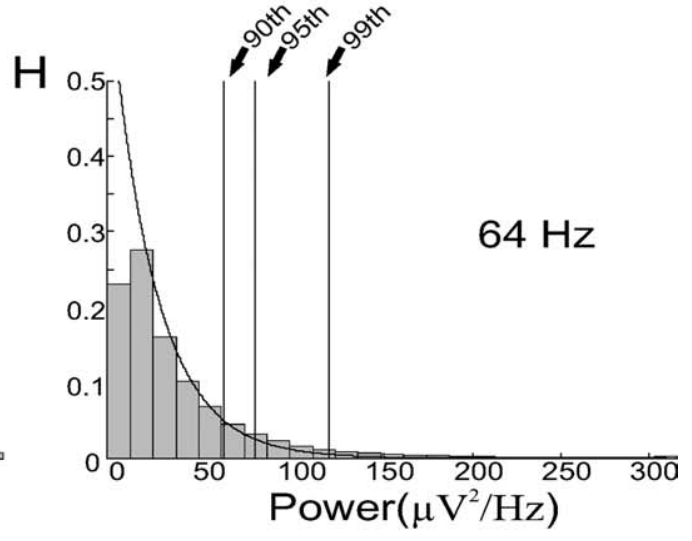

sion fit obtained from the deactivated state. Vertical lines represent the 90th, 95th, and 99th percentile thresholds (left to right). Note that for some frequencies, some power values are beyond the range of plotted here; those are summed in the right-most bin. At delta frequencies (most prominently at $1 \mathrm{~Hz}$ ), the distributions deviate substantially from the expected $\chi^{2}(2)$ but at other frequencies, the fit is reasonable, in line with the assumptions of the BOSC method. 


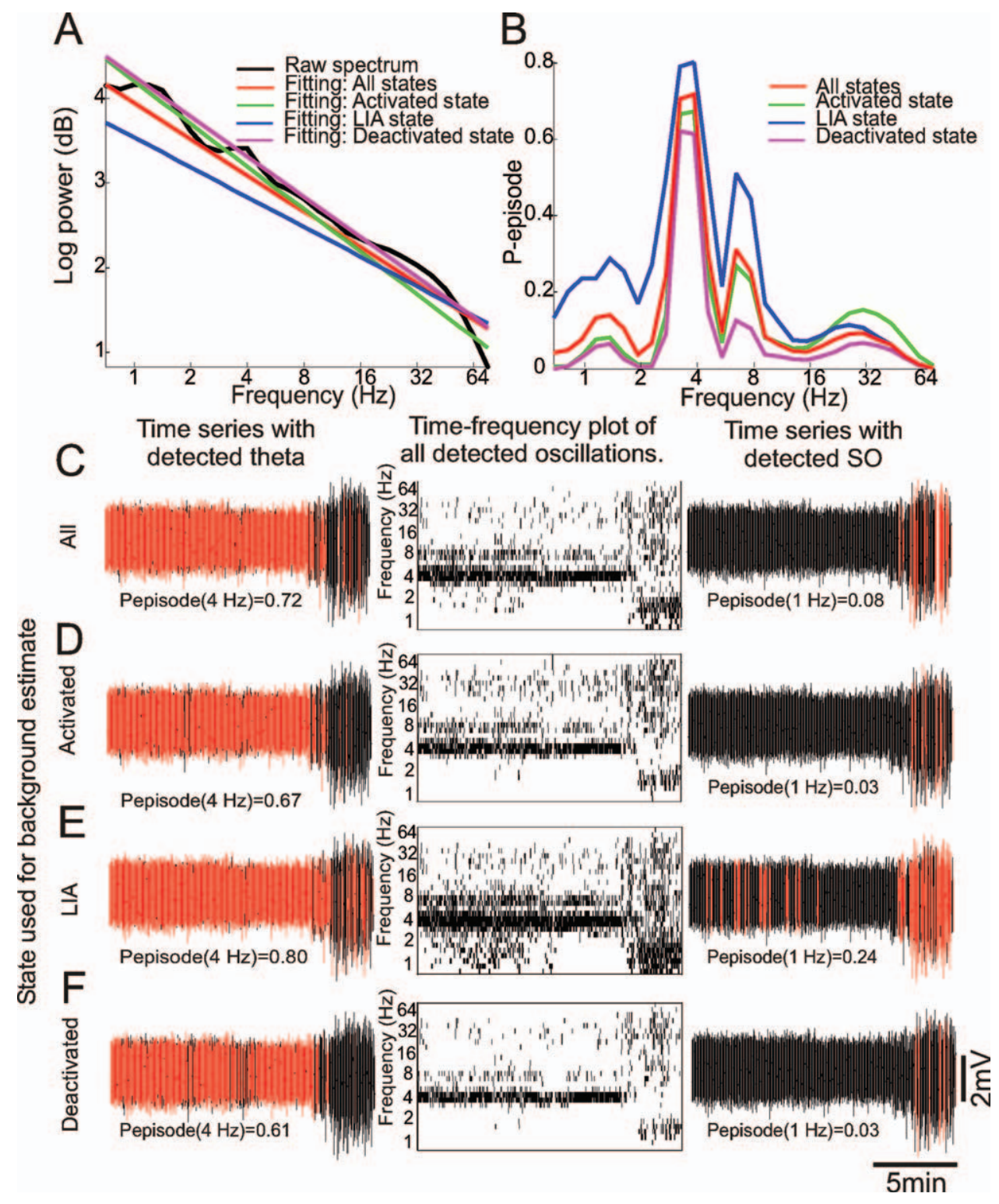

FIGURE 5. Detection of theta $(4 \mathrm{~Hz})$ and the slow $(1 \mathrm{~Hz})$ oscillations: The effects of different background power estimates within, between, and across states. A: Power spectrum from one epoch including all states with the estimated mean powers obtained from either across all states, the activated state, LIA state, or the deactivated state. B: $P_{\text {episode }}(f)$ computed across all states, using each

background estimate came from periods of LIA (Figs. 5C-F). Furthermore, a summary measure, $P_{\text {episode }}$ (defined as the proportion of time occupied by BOSC-detected oscillations), calculated using these different background spectrum estimates ranged from 0.61 to 0.80 (Figs. 5B-E). It is clear that rhythmic activity segments are indeed being detected (Fig. 6). Thus, the BOSC method was able to pull out the continuous $3-4 \mathrm{~Hz}$ theta activity, something it had not yet been tested on, with minimal sensitivity to the state used to calculate $P_{\mathrm{T}}$. It is also worth noting that a common technique, prewhitening (dividing all power values by the mean power at each frequency) would method of estimating mean background power (as in A). C-F: The raw time series (black) with the detected oscillations at $4 \mathrm{~Hz}$ (left panels) or $1 \mathrm{~Hz}$ (right panels) plotted in red and time-frequency plots of detected oscillations over the same time span (middle panels). Background estimate from all states (C), from the activated state (D), from LIA (E), and from the deactivated state (F).

only be sensitive to modulations in theta power but might completely overlook the overall presence of nearly continuous theta if that rhythmic activity is sustained throughout the entire recording segment. In addition, if correcting relative to a baseline were done with hybrid states, then the results would be highly dependent on the proportion of time occupied by theta oscillations, producing variable results across recordings.

We examined the breaks in detection of $4-\mathrm{Hz}$ oscillation during the activated period more closely by assessing whether they were due purely to amplitude modulation or to spontaneous phase resetting of the theta signal. By filtering the raw 


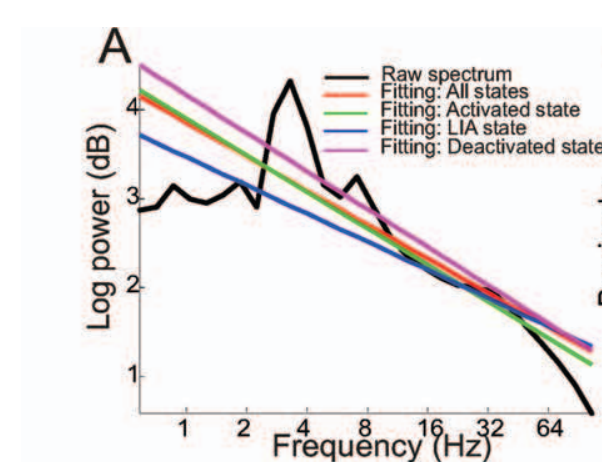

Time series with detected theta
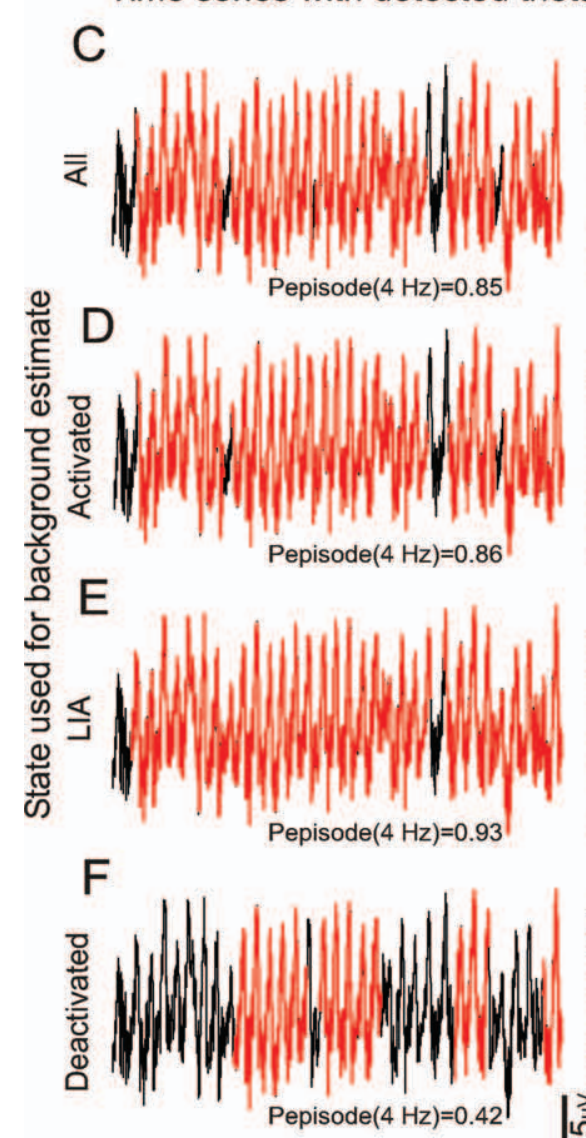

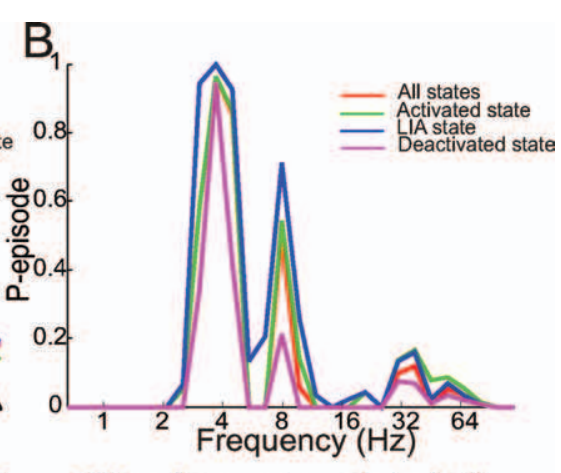

Time-frequency plot of all detected oscillations
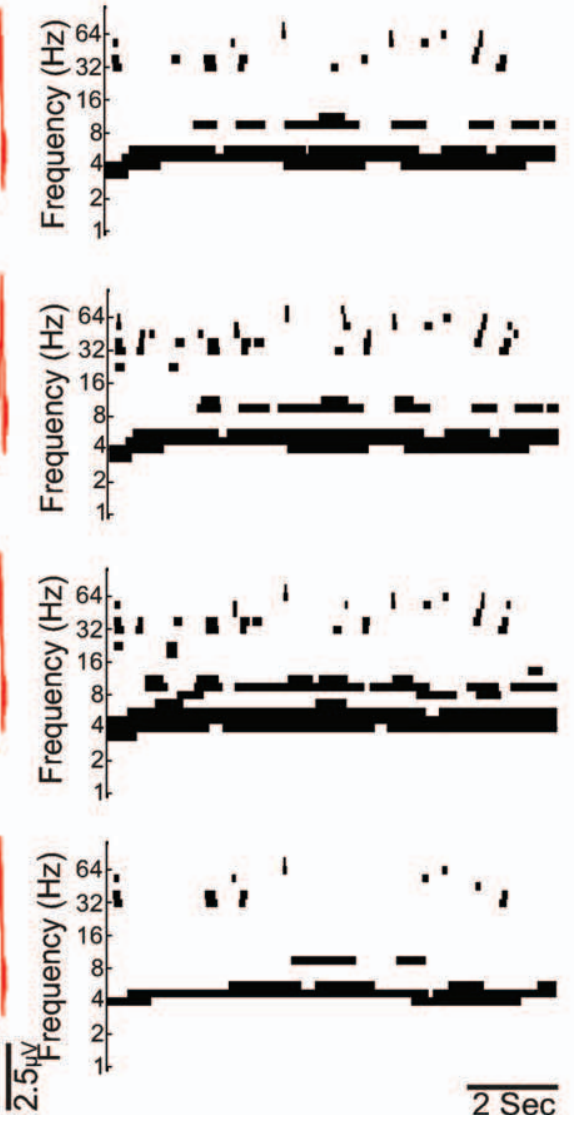

FIGURE 6. Detection of theta $(4 \mathrm{~Hz})$ oscillations during the activated state: The effects of different background power estimates within, between, and across states. A: Power spectrum from a 10-s segment of the activated state, overlaid with (linear) estimated mean background power derived from all states, the activated state, LIA, or the deactivated state. B: $P_{\text {episode }}(f)$

signal in a tight bandwidth centered at $4 \mathrm{~Hz}(3.37-4.76)$ and computing the instantaneous phase via Hilbert transform of the filtered signal (Le Van Quyen et al., 2001), we were able to evaluate both hypotheses by comparing to the results of BOSC (Fig. 7). Although time points corresponding to nondetections using BOSC were typically concomitant with decreases in the amplitude of the $4-\mathrm{Hz}$ signal (which is unsurprising based on the dependency of BOSC on amplitude), there were many examples of decreasing amplitude that were not associated with obtained with each of the background estimates. C-F: Raw, 10-s time series (black) with the detected oscillations at $4 \mathrm{~Hz}$ plotted in red (left panels) and time-frequency plots of detected oscillations (right panels). Background estimate from all states (C) from the activated state (D), from LIA (E), and from the deactivated state $(\mathrm{F})$.

nondetection (see arrows Fig. 7) that still corresponded to successful detections. By plotting the slope of the phase component of the Hilbert transform (which is constant for a consistent oscillatory process like a sine wave), we were able to visualize sudden changes in the phase which, when they were large enough, were consistently coupled to nondetected periods. This suggests that although BOSC uses power as a threshold factor, it is the consistency of the oscillation that it is critically dependent on. 


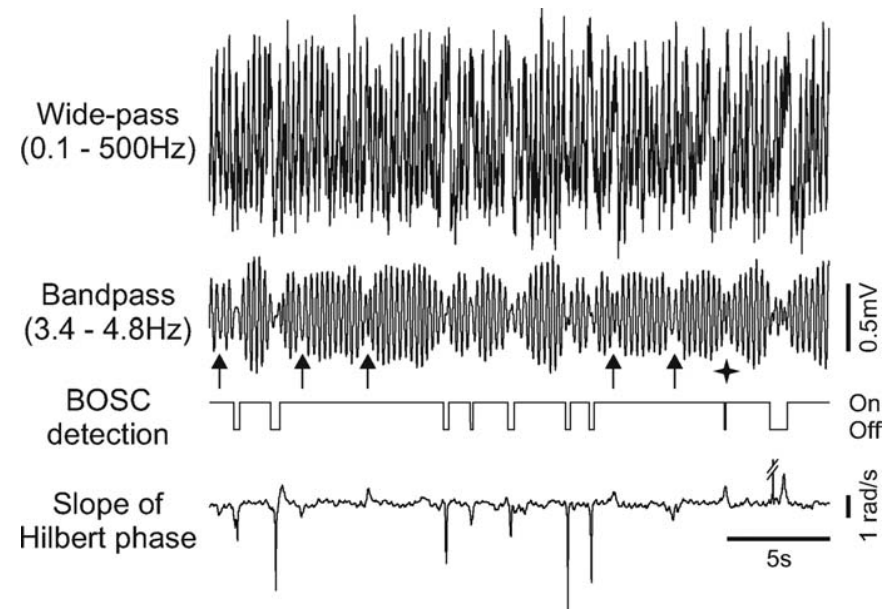

FIGURE 7. Tracking BOSC results to fluctuations in the amplitude and phase of the $4-\mathrm{Hz}$ theta component. Shown (in order from top to bottom) are the raw LFP, the $4-\mathrm{Hz}$ bandpass-filtered version, the BOSC detection results, and the slope of the phase of the filtered signal as computed from the Hilbert transform. Note that it is sudden and large changes in phase and not necessarily amplitude (see arrows) of the filtered signal that correspond to the breaks in BOSC detection. The star marks a BOSC nondetection that shows a comparable change in amplitude to the ones denoted with arrows.

\section{The BOSC Method for Detecting the Hippocampal SO}

The hippocampal SO (Isomura et al., 2006; Wolansky et al., 2006) is a novel brain state that has recently emerged as an important large-amplitude hippocampal rhythm. A major difference with theta (in addition to the fact that they are mutually exclusive based on behavioral state) is that the $\mathrm{SO}$ is intermittent and short-lasting. As shown in Figures 5 and 8, the BOSC method was able to extract sporadic SO rhythms during the deactivated state, consistent with previous reports.

When the background window was calculated across states, as well as within the deactivated state, or even from the activated state, there was a difference in the linear fit of the background power estimate (Fig. 8A). The $P_{\text {episode }}$ calculated with the different power estimates were variable at $1 \mathrm{~Hz}$ (Fig. 8B), as they were for theta in the activated state. When the background estimate was calculated from within the deactivated state, it was the most conservative, with fewer oscillatory epochs detected at all frequencies (Figs. 5C-F and 8C-F). The background estimates including other states resulted in very similar $P_{\text {episode }}(f)$ at $1 \mathrm{~Hz}$ but were quite different at other frequencies (Fig. 8B).

\section{Validation by Human Raters}

Each rater independently reviewed the same data set three times. Signals were derived from a single deactivated period that had been previously assessed by both BOSC, using the conservative within state background, and FPPA methods. Windows of 3 -s duration were randomly presented from this epoch. To assess agreement within and across raters, each set of ratings was corre- lated with every other. As well, each rating was correlated with the results of both the FPPA and BOSC methods.

In two different runs (of three trials) across 3-s segments extracted from the same time series sequence but having different samples across runs (first run $n=144$, second run $n=$ 121), raters showed a high degree of consistency across trials. The average correlations were $0.80 \pm 0.05$ and $0.92 \pm 0.02$ for the first and second runs, respectively (64 and $85 \%$ variance accounted for, respectively). Thus, experts were reasonably internally consistent across rating trials.

Inter-rater consistency was also reasonable. For the initial run (as above), the average rating agreement was high $(r=$ $0.78 \pm 0.02 ; 61 \%$ variance accounted for), and for the second run, the inter-rater correlation was again similarly high $(r=$ $0.73 \pm 0.11 ; 53 \%$ variance accounted for). Thus, experts were consistent with each other.

The consistency of experts' ratings with the FPPA and BOSC methods were substantially different. The average correspondence to the FPPA method was very low: $r=0.28 \pm$ 0.02 (7.8\% variance accounted for), whereas to the BOSC method it was just as high as the interexpert correspondence: $r=0.74 \pm 0.01$ (55\% variance accounted for). Thus, the BOSC method outperformed the FPPA method in terms of consistency with expert human raters.

For overlapping data classification segments, the agreement between BOSC and FPPA was equivalently low $(r=0.29$, $8.2 \%$ variance accounted for). Closer examination of the mismatches between BOSC and FPPA revealed two situations. The most common was a tendency for FPPA to be too liberal in classifying low bandwidth power as $\mathrm{SO}$, even when not obviously rhythmic (Fig. 9A). A less frequent occurrence was the BOSC detection of short but just threshold oscillations that were imbedded in backgrounds having less power in the lower bandwidths (Fig. 9B). During both these cases, human raters were more likely to agree with BOSC than FPPA. Given the reasonable correspondence of human expert ratings to the BOSC method, we thought it useful to assess the influence of feedback training in which raters were provided with the results of the BOSC method following each sample evaluation. Interestingly, during these trials, intrarater consistency improved to $0.89 \pm 0.05(79 \%$ variance accounted for) and in trials subsequent to the initial trial run this consistency improved to 0.93 \pm 0.04 ( $86 \%$ variance accounted for). As well, inter-rater consistency also improved to $0.89 \pm 0.02 \quad(79 \%$ variance accounted for) and in trials subsequent to the first run this further improved to $0.92 \pm 0.01$ ( $85 \%$ variance accounted for). Not surprisingly, consistency with the BOSC method itself also increased substantially: $0.91 \pm 0.03(83 \%$ variance accounted for; in trials subsequent to the first run this improved only slightly: $0.92 \pm 0.02 ; 85 \%$ variance accounted for).

\section{DISCUSSION}

This study demonstrates that the BOSC method is a suitable and versatile detection algorithm for oscillatory epochs across a 

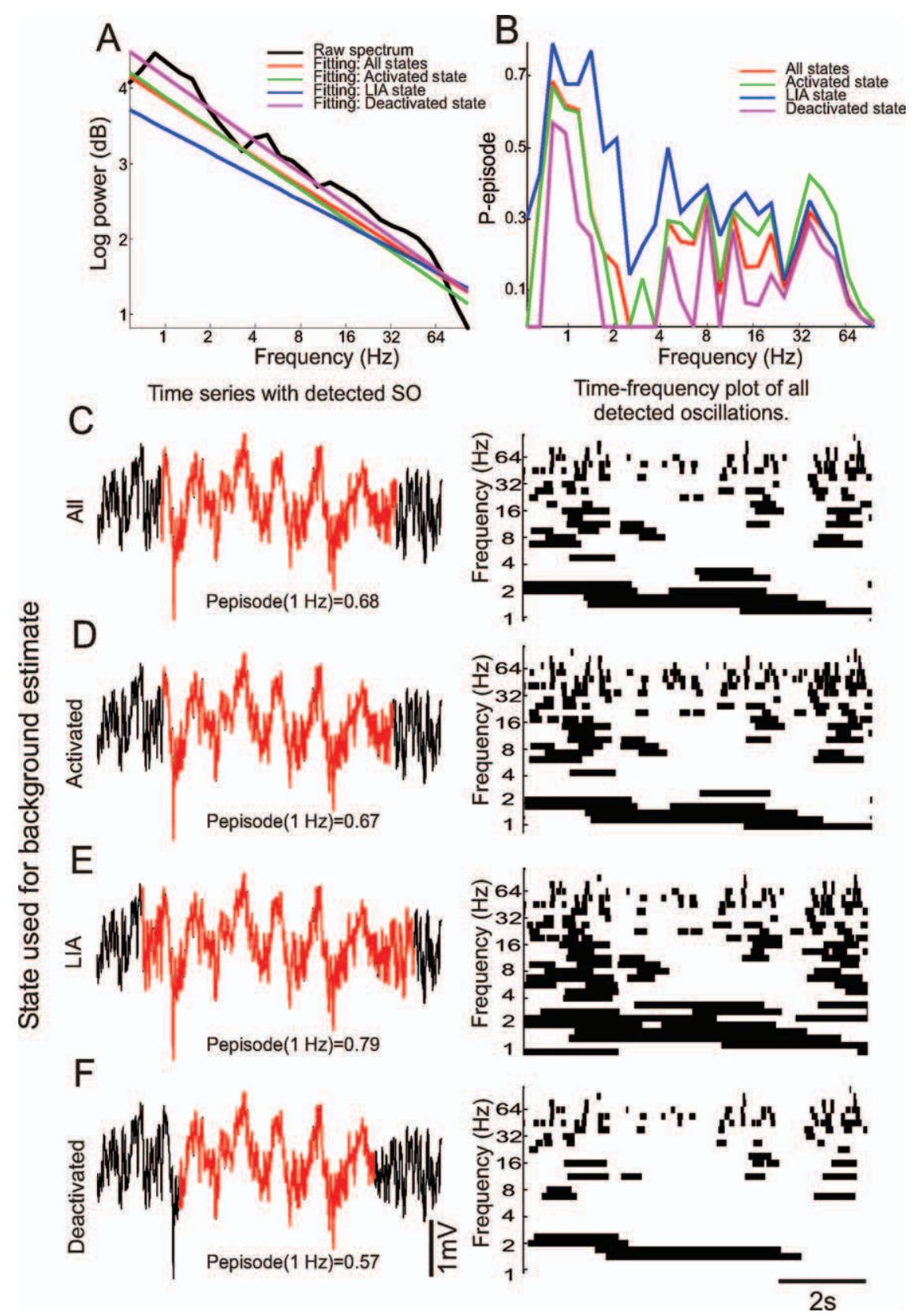

FIGURE 8. Detection of slow $(1 \mathrm{~Hz})$ oscillations during the deactivated state: The effects of different background power estimates within, between, and across states. A: Power spectrum for an 8-s second segment of the deactivated state, overlaid with (linear) estimated mean background power derived from all states,

the activated state, LIA and the deactivated state. B: The $P_{\text {episode }}(f)$ obtained with each of the background estimates. C-F: Raw, 8-s time series (black) with detected oscillations at $1 \mathrm{~Hz}$ plotted in red (left panels) and time-frequency plots of detected oscillations (right panels).

multitude of conditions. In particular, it shows that the method is able to detect both sporadic (SO) and near-continuous (theta) oscillatory trains of activity in the rat hippocampal network, even when state changes contribute to the signal on which the method's power threshold is based. This is strong evidence that the BOSC technique is robust to the influences that state changes can have on the shape of the average power spectrum. Furthermore, the detection of oscillatory epochs using BOSC, but not the Fourier peak power analytic method, was correlated well with expert ratings of the same oscillatory episodes and thus BOSC is principled, reliable, and accurate. Given the present demonstration and those prior (Whitten et al., 2011), we conclude that the BOSC algorithm is a reasonably robust method that is suitable for oscillation detection across species, frequencies, regions, tasks, and states. The advantages conferred by this method include its automation, which allows for speedy processing, and an express lack of bias since it objectively provides quantifiable values for the detection of oscillations. 


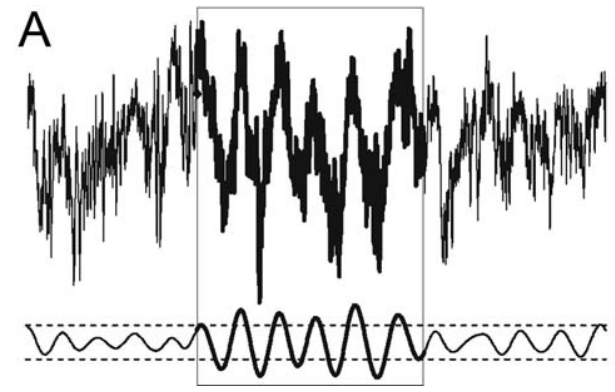

FIGURE 9. Comparison of BOSC to FPPA detection of slow $(1 \mathrm{~Hz})$ oscillations. A: An example raw (upper trace) and SO-filtered (0.84-1.19 Hz: lower trace) during the deactivated state. The dotted lines represent the statistical upper and lower limits ( $95 \%$ confidence interval) based on twice the value of the RMS of the filtered signal. The box shows a clearly defined BOSC-detected epoch of SO that was also detected with FPPA despite showing no

\section{The BOSC Method-A Versatile, Robust, and Reliable Oscillation Detection Algorithm}

Originally, the BOSC method was developed to extract sporadic oscillatory activity from recordings having a multitude of wide-band components that made detection difficult for other spectral-based methods (Caplan et al., 2001). Until now, no one has evaluated or used the BOSC method for the detection of longer runs of near-continuous oscillatory activity such as rat hippocampal theta. Although other methods may appear well suited and perhaps more appropriate for this latter type of detection, we determined that BOSC also performed well for this purpose and was useful beyond the simple detection of oscillatory activity per se. Indeed, because the BOSC measure is so sensitive to variations in both amplitude and frequency features of oscillations, we were able to consistently detect sporadic and momentary breaks in otherwise seemingly continuous trains of theta activity, which corresponded to sudden shifts in the normal progression of phase values. Thus, the BOSC method can be used in an efficient dual-mode fashion to mine data segments for both transient oscillatory activity within epochs that are mainly nonoscillatory as well as for nonoscillatory periods during epochs containing more continuous oscillatory activity. Together, the results of this evaluation speak to the versatility of the method and highlight its significance for the evaluation of neural dynamics in large datasets.

An additional important confirmation made here was that the detection of oscillations by the BOSC method was relatively insensitive to state-dependent components present in the background used for the estimated fitting. This is an important characteristic because many experiments have no prima facie control over the electrographic state during which a particular oscillation may be expressed or not. We specifically tested the resilience of the BOSC method to state changes by examining the influence of the particular background chosen (activated versus deactivated) for both types of oscillatory activity (theta versus the SO). The fact that BOSC was relatively insensitive to changes in the components of the background spectrum

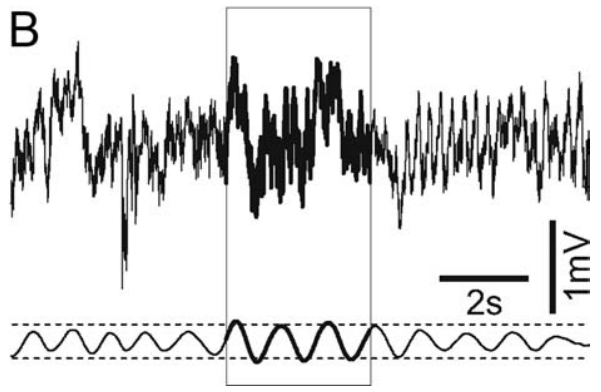

clear above-threshold rhythmic activity when visualizing the filtered trace. B: Another example raw and SO-filtered trace (as in A) during a transition from activated to deactivated state. The box shows a BOSC-detected epoch of SO that was not detected using PPA. Although the raw trace appears not to contain any SO activity, the filtered trace shows just-threshold activity for three cycles.

were in part because the assumptions about the form of the background spectrum (colored noise spectrum with $\chi^{2}(2)$-distributed wavelet power values) hold sufficiently well across the states examined here (Figs. 3 and 4). In addition, the power values one obtains due to the two types of rhythmic activity here (theta and the SO) are sufficiently high that the precise value of the power threshold is not so critical (Figs. 3 and 4), which may in part underlie the demonstrated robustness to state changes; this parallels the resilience of the BOSC method to fluctuations in and out of the alpha rhythm in humans (Whitten et al., 2011). Although relatively robust to transitions across state, the method did perform nominally better when power estimates came from within the state during which the particular oscillation was expressed.

This relative robustness of the BOSC method to state changes means one could use the method to ask research questions about state changes themselves. However, for research questions geared toward examining changes in oscillatory activity within-state, collapsing across major state changes is not optimal. Although the effects of state changes were not large (Figs. 5, 6, and 8), when possible, measures of oscillatory activity would be more precise if comparisons were made withinstate. It would therefore be prudent to account for the current state if known. If state transitions were not known, a first pass with BOSC, detecting oscillations at all frequencies using the entire background signal as a power estimate, could be done. State changes could then be identified by either plotting the power at the target frequencies over time as was done here or through visual inspection by a trained observer. Once state changes are identified, the BOSC method could be reapplied, using background estimates to derive the $P_{\mathrm{T}}$ values within-state only.

Using the best-case detection background for the SO, we also showed that the method was at least as consistent with expert human raters as were the raters themselves. The low correspondence of human raters to the FPPA method for the same dataset highlights the superiority of the BOSC method even when not considering its other advantages (especially 
automation). In fact, the correspondence within and between raters and between raters and the BOSC method itself was improved to $\sim 90 \%$ in subsequent trials in which the raters were provided with feedback postrating regarding the BOSC classification. This suggests that although human raters still have some quantifiable bias, with BOSC feedback training they can become almost as consistent as the method itself.

In sum, our findings show for the first time that the BOSC method can be used in nonhuman electrophysiological recordings to detect sustained and transient episodes of oscillatory activity. The BOSC method was able to detect SO during the deactivated state as previously shown (Wolansky et al., 2006). Although the background state used for SO detection did affect the total number of detected epochs, the results were still qualitatively similar and the state-specificity remained. Another major advantage of this method is its automation allowing for one to perform oscillation detection with minimal subjective involvement and maximal efficiency. As one can use the method to plot detected oscillations over the raw trace, the careful researcher can also cross-check the results ensuring the accuracy of the method. This gives researchers a new and objective method to identify oscillatory activity from human and nonhuman electrophysiological recordings and allows one to compare oscillatory epochs across different species, brain states, and tasks.

\section{Acknowledgments}

The authors thank Leanna Cruikshank and Arne Ekstrom for valuable feedback on the manuscript, Chris Madan for technical assistance and Kingsley Chan and Caitlin Hughes for data collection.

\section{REFERENCES}

Berger H. 1929. Uber das elektrenkephalogramm des meschen. Arch Psychiatr Nervenkr 87:527-570.

Bland BH. 1986. The physiology and pharmacology of hippocampal formation theta rhythms. Prog Neurobiol 26:1-54.

Bland BH, Oddie SD. 2001. Theta band oscillation and synchrony in the hippocampal formation and associated structures: the case for its role in sensorimotor integration. Behav Brain Res 127:119-136.

Buzsaki G. 1989. Two-stage model of memory trace formation: a role for "noisy" brain states. Neuroscience 31:551-570.

Buzsaki G. 2002. Theta oscillations in the hippocampus. Neuron 33: 325-340.

Buzsaki G, Draguhn A. 2004. Neuronal oscillations in cortical networks. Science 304:1926-1929.

Caplan JB, Glaholt MG. 2007. The roles of EEG oscillations in learning relational information. Neuroimage 38:604-616.

Caplan JB, Madsen JR, Raghavachari S, Kahana MJ. 2001. Distinct patterns of brain oscillations underlie two basic parameters of human maze learning. J Neurophysiol 86:368-380.

Caplan JB, Madsen JR, Schulze-Bonhage A, Aschenbrenner-Scheibe R, Newman EL, Kahana MJ. 2003. Human theta oscillations related to sensorimotor integration and spatial learning. J Neurosci 23: 4726-4736.

Chrobak JJ, Lorincz A, Buzsaki G. 2000. Physiological patterns in the hippocampo-entorhinal cortex system. Hippocampus 10:457-465.
Clement EA, Richard A, Thwaites M, Ailon J, Peters S, et al. 2008. Cyclic and Sleep-Like Spontaneous Alternations of Brain State Under Urethane Anaesthesia. PLoS ONE 3(4):e2004. doi:10.1371/ journal.pone.0002004.

Dickson CT. 2010. Ups and downs in the hippocampus: the influence of oscillatory sleep states on "neuroplasticity" at different time scales. Behav Brain Res 214:35-41.

Ekstrom AD, Caplan JB, Ho E, Shattuck K, Fried I, Kahana MJ. 2005. Human hippocampal theta activity during virtual navigation. Hippocampus 15:881-889.

Freunberger R, Fellinger R, Sauseng P, Gruber W, Klimesch W. 2009. Dissociation between phase-locked and nonphase-locked alpha oscillations in a working memory task. Hum Brain Mapp 30: 3417-3425.

Hasselmo ME. 2005. What is the function of hippocampal theta rhythm?-Linking behavioral data to phasic properties of field potential and unit recording data. Hippocampus 15:936-949.

Isomura Y, Sirota A, Ozen S, Montgomery S, Mizuseki K, Henze DA, Buzsaki G. 2006. Integration and segregation of activity in entorhinal-hippocampal subregions by neocortical slow oscillations. Neuron 52:871-882.

Klimesch W, Sauseng P, Hanslmayr S. 2007. EEG alpha oscillations: the inhibition-timing hypothesis. Brain Res Rev 53:63-88.

Le Van Quyen M, Foucher J, Lachaux J, Rodriguez E, Lutz A, Martinerie J, Varela FJ. 2001. Comparison of Hilbert transform and wavelet methods for the analysis of neuronal synchrony. J Neurosci Methods 111:83-98.

Marshall L, Born J. 2007. The contribution of sleep to hippocampusdependent memory consolidation. Trends Cogn Sci 10:442-450.

Marzano C, Ferrara M, Mauro F, Moroni F, Gorgoni M, Tempesta D, Cipolli C, De Gennaro L. 2011. Recalling and forgetting dreams: theta and alpha oscillations during sleep predict subsequent dream recall. J Neurosci 31:6674-6683.

Percival DB, Walden AT. 1993. Spectral Analysis for Physical Applications: Multitaper and Conventional Univariate Techniques. Cambridge: Cambridge University Press.

Plett M. 2000. Ultrasonic Arterial Vibrometry with Wavelet Based Detection and Estimation. Seattle, WA: University of Washington.

Schall KP, Kerber J, Dickson CT. 2008. Rhythmic constraints on hippocampal processing: State and phase-related fluctuations of synaptic excitability during theta and the slow oscillation. J Neurophysiol 99:888-899.

Schiff SJ, Aldroubi A, Unser M, Sato S. 1994. Fast wavelet transformation of EEG. Electroencephalogr Clin Neurophysiol 91:442-455.

Schlesinger MF, West BJ. 1988. 1/f versus 1/f noise. In: Stanley EH, Ostrowsky EH, editors. Random Fluctuations and Pattern Growth: Experiments and Models. Dordrecht: Kluwer. pp 320324.

van Vugt MK, Sederberg PB, Kahana MJ. 2007. Comparison of spectral analysis methods for characterizing brain oscillations. J Neurosci Methods 162:49-63.

Vanderwolf CH. 1969. Hippocampal electrical activity and voluntary movement in the rat. Electroencephalogr Clin Neurophysiol 26:407-418.

Watrous AJ, Fried I, Ekstrom AD. 2011. Behavioral Correlates of Human Hippocampal Delta and Theta Oscillations During Navigation. J Neurophysiol 105:1747-1755.

Whitten TA, Hughes AM, Dickson CT, Caplan JB. 2011. A better oscillation detection method robustly extracts EEG rhythms across brain state changes: The human alpha rhythm as a test case. Neuroimage 54:860-874.

Wolansky T, Clement EA, Peters SR, Palczak MA, Dickson CT. 2006. Hippocampal slow oscillation: A novel EEG state and its coordination with ongoing neocortical activity. J Neurosci 26:6213-6229.

Worden MS, Foxe JJ, Wang N, Simpson GV. 2000. Anticipatory biasing of visuospatial attention indexed by retinotopically specific alpha-band electroencephalography increases over occipital cortex. J Neurosci 20:RC63. 